\title{
Developing Connections among B Lymphocytes and Deregulated Pathways in Autoimmunity
}

\author{
Moncef Zouali ${ }^{1,2}$ and Gregory Tsay, ${ }^{3,4}$
}

${ }^{1}$ National Institute of Health and Medical Research (Inserm), Paris, France; ${ }^{2}$ Paris Diderot University, Sorbonne Paris-Cité, Paris, France; ${ }^{3}$ Division of Immunology and Rheumatology, Department of Internal Medicine, China Medical University Hospital, Taichung, Taiwan; and ${ }^{4}$ College of Medicine, China Medical University, Taichung, Taiwan

\begin{abstract}
Immunologists have long investigated B lymphocytes as solely antibody-producing cells. With further studies, it became clear that B cells can exert a variety of functions within the immune system and beyond. As a result, B cells are considered promising targets for immunotherapy in a variety of disorders. Recently, experts in B cell biology and autoimmunity convened to discuss important stepping stones to decipher the complexity of B lymphocyte-mediated pathways in autoimmune diseases. Twenty-five years ago, the first report describing the clinical efficacy of depleting B lymphocytes in autoimmune diseases was published (1). This also marked organization of the first International Conference on B Cells and Autoimmunity, held in 2001 in Bergen, Norway. Since then, the credentials of B cells as essential players in autoimmune disease have been well established. For its sixth edition, this conference series settled along the shores of Sun Moon Lake in the heart of Taiwan on August 16-18, 2016, to view and discuss recent advances in different facets of B cell biology and put them in the perspective of understanding autoimmunity and designing effective immunointervention strategies. Following the tradition established in 2001, the conference was a forum where basic immunologists and their clinically trained colleagues met to discuss hot topics of autoimmunity research. Speakers from four continents discussed some of their new data and insights and brought considerable excitement to the conference in the refreshingly beautiful and elegant landscape of Sun Moon Lake and its indescribable charm.
\end{abstract}

Online address: http://www.molmed.org

doi: 10.2119/molmed.2016.00206

\section{B CELL TOLERANCE AT THE GERMINAL CENTER CHECKPOINT}

Secondary lymphoid organs harbor specialized microenvironments, termed germinal centers (GCs), that generate high-affinity, long-lived antibody-forming cells and memory B cells. In the absence of deliberate immunization or infection, GCs can spontaneously develop (SptGCs). In autoimmune disease, such as systemic lupus erythematous (SLE), inappropriate maintenance of $\mathrm{B}$ cell tolerance at the GC checkpoint is believed to give rise to autoreactive B cells in Spt-GCs, and it is possible that cooperative interactions between an innate immune receptor, such as a Toll-like receptor (TLR), and the antigen $B$ cell receptor allow B lymphocytes to reach an activation threshold that overcomes immune tolerance. For example, production of type I interferon (IFN) is regarded as playing an important role in SLE pathogenesis and depends on the recognition of viral and self nucleic acids by cellular sensors, such as the endosomal TLR7, with experimental deficiency of the $T \operatorname{lr} 7$ gene leading to complete abrogation of

Address correspondence to Moncef Zouali, Inserm U1 132, Centre Viggo Petersen, Hôpital Lariboisière, 2, rue Ambroise Paré, 75475 Paris Cedex 10, France. Phone: + (33) 1499563 28; Fax: + (33) 1499584 52; E-mail: moncef.zouali@wanadoo.fr Submitted October 4, 2016; Accepted for Publication October 4, 2016; Published Online (www.molmed.org) October 5, 2016.

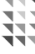

Feinstein Institute for Medical Research Northwell Health

lupus disease. Previously, TLR7 was found to exert B cell-intrinsic effects in promoting spontaneous GC and plasmablast development (2). Ziaur SM Rahman (Pennsylvania State University, USA) identified B cell-intrinsic TLR7 signaling as a prerequisite to Spt-GC formation. In autoimmune B6.Sle1b mice, TLR7 deficiency in B cells rendered the rodents unable to develop Spt-GCs and led to markedly decreased autoantibody production (2). Strikingly, B6.Sle1b.yaa mice, which express an extra copy of the tlr7 gene, had increased Spt-GC, T follicular helper (Tfh) cells and autoantibody responses. Treatment of B6.Sle1b mice with a TLR7 agonist resulted in highly elevated GC, Tfh, and autoantibody responses, along with increased nephritis and a female bias, features that make these mice an attractive model to study mechanisms of TLR7-driven autoimmune B cell responses and SLE-like autoimmunity. These observations suggest an essential role for TLR7 in promoting Spt-GC and autoimmune responses (3). 
Rahman's data also indicate a critical B cell-intrinsic role of interferon $\gamma$ receptor (IFN $\gamma \mathrm{R})$ signaling in accentuating Spt-GCs and autoimmune responses in B6.Sle1b mice (4). They suggest that IFN $\gamma R$ and STAT1 signaling control Spt-GC and Tfh formation by driving T-bet expression and IFN $\gamma$ production by B cells. Global or B cell-specific IFN $\gamma$ R deficiency in autoimmune B6.Sle1b mice leads to significantly reduced Spt-GC and Tfh responses and results in diminished autoantibody production reactivity compared with B6.Sle1b mice. The fact that proliferation and differentiation of DNA-reactive B cells into a GC B cell phenotype require $B$ cell-intrinsic IFN $\gamma \mathrm{R}$ signaling suggests that IFN $\gamma \mathrm{R}$ signaling regulates GC B cell tolerance to nuclear self-antigens. However, the IFN $\gamma \mathrm{R}$ deficiency does not affect GCs, Tfh, and antibody responses against $\mathrm{T}$ cell-dependent foreign antigens, indicating that IFN $\gamma \mathrm{R}$ signaling regulates autoimmune, but not protein antigen-driven GC and Tfh responses. Thus, these data define a novel B cell-intrinsic TLR7 and IFN $\gamma$ R-STAT1 signaling pathway specific to Spt-GC development and autoimmunity. It will be important to determine if this pathway, which seems to promote aberrantly regulated Spt-GC response in SLE, can be targeted by pharmacological intervention to treat systemic autoimmunity.

\section{PATHOGENIC AUTOANTIBODIES OF THE IGE ISOTYPE}

Characterization of the isotypes of autoantibodies found in patients with autoimmune disease has been the focus of much investigation. It can provide clues for understanding disease pathogenesis (5) and potential biomarkers, because autoantibodies can exist in the sera of patients several years prior to the onset of clinical disease (6). Early studies disclosed that antinuclear antibodies of the IgE class are present in lupus patients and that they could be of pathogenetic importance (7). Miguel Sanjuan (MedImmune, Gaithersburg, MD, USA) showed that, in patients with SLE, the levels of dsDNA-specific IgE autoantibodies correlate with disease severity. Deposits of IgE were also found in the kidneys of patients with lupus nephritis. Notably, immune complexes formed by $\operatorname{IgE}$ specific for DNA induced the release of much IFN $\alpha$ upon binding to FceRI at the cell surface and, subsequently, to TLR9 at the lumen of phagosomes in plasmacytoid dendritic cells (pDCs). These findings provide a novel mechanistic link for the participation of $\operatorname{IgE}$ autoantibodies in autoimmunity (8). In some kidney biopsies from lupus patients, pDCs and B cells were also found in close proximity, suggesting possible cell-cell interactions. In vitro, $\mathrm{pDC}$ activated with IgE immune complexes were capable of driving B cell expansion and plasma cell differentiation. Importantly, IgE immune complexes induced plasma cell differentiation to a similar degree as that observed in response to IL-21, a pleiotropic cytokine known to induce B cell activation and differentiation, which is widely regarded as a potent driver of humoral immune response in humans. Furthermore, there was a significant increase in the frequency and number of plasmablasts in response to IgE immune complexes compared with IL-21 co-stimulation. The ability of IgE to induce pDC-dependent plasma cell differentiation relied mainly on soluble factors and on antibodies that blocked the type I IFN receptor.

In SLE patients, the levels of circulating anti-DNA IgG autoantibodies were found to be at least one order of magnitude higher than their IgE counterparts, and seropositivity for anti-DNA IgE autoantibodies was always accompanied by the presence of anti-DNA IgG autoantibodies (8). To determine the contribution of IgE autoantibodies to the pathogenic secretion of IFNs in SLE, Sanjuan described in vitro experiments revealing that IgE synergizes with IgG in triggering secretion of IFN $\alpha$ and other proinflammatory mediators. The synergy was mechanistically explained by an enhanced uptake of dsDNA-containing immune complexes resulting from the combining action of simultaneously activating Fc $\gamma$ RII $\alpha$ and FceRI at the cell surface of $\mathrm{pDC}$. This was even the case when the concentration of $\operatorname{IgE}$ in the immune complexes was several orders of magnitude lower than that of $\operatorname{IgG}$, a scenario that resembles what is observed in patients. Finally, a blocking anti-IgE antibody was able to significantly reduce the production of IFN $\alpha$ in peripheral blood mononuclear cells exposed to SLE serum, demonstrating that IgE autoantibodies contribute significantly to the inflammatory responses that underlie the disease. Thus, these experiments reveal that $\operatorname{IgE}$ autoantibodies specific for DNA have the capacity to induce potent humoral responses and represent a novel mechanism that could support the generation of pathogenic autoantibodies in lupus patients. They establish a mechanistic link between anti-DNA IgE autoantibodies, pDCs and type I IFNs, whose unabated secretion is central to SLE, a concept that has now been clinically validated by blocking the type I IFN receptor in a phase 2 proof-of-concept study using the anti-IFN $\alpha$ receptor monoclonal anifrolumab antibody (9).

\section{TISSUE-RESIDENT LYMPHOCYTES}

Having evolved to generate a huge antigen-specific repertoire and mount $\mathrm{T}$ cell-dependent responses and longterm memory, the B lymphocyte is a central player in the adaptive branch of immune defense. However, accumulating evidence indicates that B-1a cells of the peritoneal cavity and marginal zone (MZ) B cells of the spleen also can have innate-like immune functions (10). Even though B-1a cells can peak rapidly to cell cycle and are excellent antigenpresenting cells, the potential interactions between T cell help and B-1a cells have not been well addressed. Tae Jin Kim (Sungkyunkwan University, Suwon, South Korea) presented data revealing the presence of a subset of peritoneal $\mathrm{CD} 4^{+} \mathrm{T}$ cells with a strong capability to boost B-1a cells. In these studies, B-1a cells had a strong tendency to form conjugates with $\mathrm{CD} 4^{+} \mathrm{T}$ cells, which resulted in production of increased amounts of antibodies compared to unconjugated 
B-1a cells (11). In the peritoneal and pleural cavities of the mice, memory phenotype $\mathrm{CD}^{+} \mathrm{T}$ cells (characterized as $\mathrm{CD} 44^{\text {high }} \mathrm{CD} 2 \mathrm{~L}^{\text {low }} \mathrm{CXCR}^{+}$integrin $\alpha 4 \beta 1^{+}$) were observed. Upon stimulation with phorbol myristate acetate (PMA) and ionomycin, these cells rapidly secreted Th1-type cytokines, such as IFN- $\gamma$, TNF- $\alpha$, and IL-2. Notably, development of the "memory phenotype" $\mathrm{CD} 4^{+} \mathrm{T}$ cells occurred spontaneously and early in the neonatal period. These cells showed stem cell-like properties characteristic of Tfh, such as expression of PD-1, ICOS, IL-21 and CXCR5. Their B cell helper activity was confirmed in vivo and in vitro. Upon adoptive transfer into $R a g 1^{-/}$mice, they enhanced B-1a cell production of IgM antibodies much more potently than other subsets of $\mathrm{CD}^{+} \mathrm{T}$ cells. Kim suggested that these cells are innate-like CD $4^{+}$ $\mathrm{T}$ cells with a dual capacity to support humoral and cellular immunity. Together with related observations (12), it seems that rapid activation of innate-like $B$ cells, including marginal zone B and B-1a cells, can be supported by innate-like $\mathrm{CD}^{+} \mathrm{T}$ cells, such as NKT cells and peritoneal innate-like $\mathrm{CD} 4^{+} \mathrm{T}$ cells. These characteristics are reminiscent of "tissue-resident cells" that represent the innate and adaptive branches of immunity and differ in their distribution in nonlymphoid tissues, yet exhibit common "innate"-like properties (13). For example, a combination of approaches has revealed that tissue-resident, "unconventional" or "innate-like" $\mathrm{T}$ cells express $\mathrm{T}$ cell receptors of limited diversity and recognize antigens in the context of nonclassical, nonpolymorphic MHC-like molecules (14). It is possible that tissue-resident cells, such as tissue-resident memory $\mathrm{T}$ cells, in concert with other cell types, allow multifaceted protection of tissue function upon diverse inflammatory threats. It will be important to decipher the potential role of such cells in autoimmune diseases.

Meanwhile, the role of B-1a cells has been well studied in models of atherosclerosis. The disease is initiated by cholesterol entry into arteries, triggering chronic immune-inflammatory lesions in the vessels. Rupture of vulnerable atherosclerotic lesions initiates thrombotic occlusion of vital arteries, precipitating heart attacks and strokes, which remain major killers despite therapeutic use of statins to lower blood cholesterol levels. Ban-Hock Toh (Monash University, Clayton, Australia) showed that, whereas peritoneal B-1a cells are atheroprotective, conventional B-2 B cells are atherogenic in experimental models $(15,16)$. Reduction of B-2 cells by administration of monoclonal antibody to CD20 or BAFF receptor and inactivation of the gene encoding BAFF receptor ameliorates atherosclerosis development in mice. One mechanism by which B-2 cells could favor atherosclerosis development is secretion of the proinflammatory cytokine TNF- $\alpha$, promoting abundant apoptosis, formation of large necrotic cores and inflammation in atherosclerotic lesions. By contrast, peritoneal B-1a cells would protect against atherosclerosis development by secretion of polyclonal natural IgM that scavenges apoptotic cells and oxidized low-density lipoproteins, thereby reducing apoptosis, necrotic cores and inflammation in atherosclerotic lesions. These atheroprotective effects are reproduced by stimulation of B-1a cells by administration of apoptotic cells or liposomes containing phosphotidylserine, a membrane lipid abundant on the surface of apoptotic cells (15). Thus, experimental studies of atherosclerosis in mouse models have shown that reduction of atherogenic B-2 cells and/or activation of atheroprotective B-1a cells protects against atherosclerosis development.

The potential of such observations for clinical translation to reduce the risk of death from heart attack and stroke remains under investigation. In human atheroma, a local B cell differentiation process was found to take place in arterial walls, and adventitial oligoclonal resident $B$ cells of atherosclerotic patients were mainly mature B-2 cells that lacked markers of terminal differentiation to plasma cells (17). Even in patients with aortic stenosis, which shares several similarities with atherosclerosis, B cells were present within aortic valves. The $B$ cells express BAFF-R, and their number was associated with valve calcification and maximum transvalvular gradient (18). It is possible that increasing numbers of B cells within aortic valves may accelerate inflammation and thus potentiate the progression of atherosclerosis. To further understand the pathophysiology of human atheroma, additional studies are required to define the properties of resident B cells in human arterial lesions

\section{TOLL-LIKE RECEPTOR-INDUCED SIGNALING IN B CELLS}

Like other complex disorders, autoimmune diseases benefit from genome-wide association studies that have revolutionized their genetics. These hypothesis-free approaches have consistently led to disease associations with B cell-specific genes, such as the adaptor $B$ cell scaffold with ankyrin repeats 1 (BANK1), further underlining the view that B lymphocytes are key players in autoimmune disease. BANK1 is primarily expressed in B cells and, to a lesser extent, myeloid cells and pDCs. BANK1 binds to and is phosphorylated by the Src-family of tyrosine kinases LYN and BLK, and interaction with the latter is required for BANK1 to bind phospholipase $C \gamma 2$ (PLC $\gamma 2$ ). In humans, the $B A N K 1$ gene has been associated with rheumatoid arthritis, systemic sclerosis and SLE (19). Among the three BANK1 single-nucleotide polymorphisms associated with SLE, two have been reproduced in multiple genome-wide association studies. Alternative splicing can lead to two dominant isoforms, namely a full-length BANK1 and an exon 2-deleted ( $\triangle \mathrm{BANK} 1$ ) isoform. Since exon 2 is critical for binding to $\mathrm{IP}_{3} \mathrm{R}$ (channels that most often mediate $\mathrm{Ca}^{2+}$ release from intracellular stores), the $\triangle \mathrm{BANK} 1$ isoform dampens $B$ cell signaling. Hence it has been suggested that the $\triangle \mathrm{BANK1}$ isoform may serve as a dominant negative regulator. In vitro, the two isoforms impart differential functional properties. To test the 
hypothesis that the full-length BANK1 isoform, but not the truncated isoform, can promote B cell activation and disease, Chandra Mohan (University of Houston, TX, USA) reported generation of two novel strains of transgenic mice, one bearing the full-length isoform of human BANK1 and one bearing the truncated human BANK1, both on a healthy C57BL/ 6 background driven by a CD19 promoter. Compared to the C57BL/ 6 mice and the transgenics bearing the truncated BANK1, mice that were transgenic for the full-length isoform of human BANK1 exhibited cellular and serological evidence of autoimmunity, including elevated anti-DNA antibodies, activated B cells, marginal zone B cells, B-1a cells and plasma cells, particularly in females. Gene microarray analyses and cell signaling studies revealed that $B$ cells from these mice significantly hyperexpressed a set of molecules that have previously been implicated in humoral autoimmunity. Collectively, the studies suggest that BANK1 activation together with a female milieu interplay to upregulate specific molecular pathways within $B$ cells, and these, in turn, may drive systemic humoral autoimmunity.

Additional evidence to support this conclusion comes from a recent study that investigated the effects of BANK1 in TLR7 signaling using the B6.Sle1.yaa mouse, a lupus model that develops disease through exacerbated TLR7 expression. The results suggest that BANK1 regulates TLR7-induced signaling pathways in B cells (20). It will be important to identify the proteins interacting with BANK1 downstream of TLR7, and to understand how BANK1 might work with other predisposing genes to cause lupus disease.

\section{MICRORNA CONTROLLING B CELL TOLERANCE}

The mechanisms that underlie production of autoreactive B cells and their escape from self-tolerance remain a focus of investigation. Patients with rheumatoid arthritis, type 1 diabetes or SLE show alterations of both central and peripheral B cell-tolerance checkpoints, and a number of signaling abnormalities that can lead to B cell hyperactivity have been described (21). For example, the phosphoinositide 3-kinase (PI3K) pathway has come to the forefront as an important signaling node in B cell differentiation and function and associated molecular events, such as V(D) $\mathrm{J}$ recombination and class switch recombination. PI3K is a lipid kinase that acts on membrane phosphatidylinositol (PtdIns) $(4,5) \mathrm{P} 2$ to produce PtdIns $(3,4,5)$ P3. In mice, unrestrained PI3K signaling leads to loss of B cell tolerance, and in humans, enhanced PI3K activation has been reported in approximately $70 \%$ of SLE patients. Counterregulation of PI3K activity is achieved by the inositol phosphatase and tensin homolog (PTEN) and SH2-containing inositol phosphatase (SHIP). PtdIns $(3,4,5) \mathrm{P} 3$ and perhaps PtdIns $(3,4) \mathrm{P} 2$ are substrates for PTEN, which has emerged as a key functional antagonist to PI3K (22). In the clinic, PTEN mutation is associated with several types of human tumors. In mice, deficiency of PTEN leads to a lupus-like autoimmune disease with nephritis, pulmonary inflammatory infiltration and lymphoid hyperplasia, suggesting that PTEN plays a beneficial role in retarding SLE development. Lidan Zhao (Peking Union Medical College Hospital, Beijing, China) described studies revealing that all SLE B cell subsets, except for memory B cells, exhibit decreased PTEN expression compared with B cells from healthy controls. The decreased PTEN expression was identified in patients with newly diagnosed, untreated SLE, and PTEN protein levels correlated with disease activity and nephritis (23). Importantly, SLE B cells were found to express increased levels of microRNA-7, -21 and -22 , and these downregulated the expression of PTEN. Additionally, a microRNA-7 antagomir corrected PTEN-related abnormalities in SLE B cells in a PTEN-dependent manner. Support for these expression-profiling studies showing deregulation of microRNA in cells from SLE patients comes from a study of an in vivo functional screen of microRNA in mice. microRNA-148a was found to protect immature murine B cells from apoptosis by suppressing expression of the autoimmune suppressor Gadd $45 \alpha$, the proapoptotic factor Bim and the tumor suppressor PTEN (24). It is possible that, in addition to genetic predisposition, upregulation of microRNA, which was reported in lymphocytes from patients with SLE, might also underlie the impaired removal of developing autoreactive B cells. Because mice haploinsufficient for either Bim or PTEN develop severe autoimmune manifestations, and since anergy, another mechanism of B cell receptor-mediated tolerance, is deficient in the absence of functional Bim or PTEN, upregulation of microRNA could promote autoimmune conditions through downregulation of Bim and/or PTEN. Inasmuch as defective microRNA regulation of PTEN contributes to B cell hyperresponsiveness in SLE patients and experimental animals, it could represent a new target for therapeutic intervention.

\section{B CELLS IN A SPONTANEOUSLY DEVELOPING BRAIN DISEASE}

Multiple sclerosis (MS) is commonly considered an autoimmune demyelinating disease, where myelin-reactive $T$ cells enter the central nervous system (CNS) and drive the inflammatory changes that ultimately create degenerative MS plaque. Less consideration has been given to the role of $\mathrm{B}$ cells in lesional pathogenesis. Hartmut Wekerle (Max Planck Institute of Neurobiology, Martinsried, Germany) summarized data indicating that B cells are regular components of immune infiltrates in early active MS lesions, and raised questions regarding the mechanisms that lead to recruitment of autoreactive B cells in spontaneously developing brain disease. To further understand the role of B cells, Wekerle described two transgenic mouse models that spontaneously develop encephalomyelitis and are based on cooperation between autoreactive $\mathrm{T}$ and B cells. The OSE mouse is a doubletransgenic C57BL/ 6 mouse with 
myelin oligodendrocyte glycoprotein (MOG)-specific T cells exposing a MOG-specific T cell receptor along with a knocked-in MOG-specific H-chain in its $\mathrm{B}$ cells. The RR mouse is a single-transgenic SJL/J mouse with a MOG-reactive T cell receptor expressed on over $70 \%$ of $\mathrm{CD} 4$ $\mathrm{T}$ cells. In both OSE and RR mice, disease development critically depends on the presence of an intact intestinal microbiota (25). Germ-free animals fail to develop encephalomyelitis, but when colonized with fecal samples, disease appears promptly. In these models, B cells have distinct roles. In OSE mice, they preferentially bind MOG antigen even in very high dilutions, concentrate, process and present it to cognate $\mathrm{T}$ cells, thus amplifying the autoimmune response process. In contrast, in RR mice, their role is restricted to the production of demyelinating autoantibodies.

B cells can contribute to autoimmune development by a variety of mechanisms, including secreting injuring autoantibodies, transporting autoantigen from tissue to secondary immune organs, presenting autoantigen to T cells, and secreting cytokines that condition the lesional tissue milieus. In MS, the persistence of oligoclonal bands that form a highly characteristic pattern over years suggests that the brain parenchyma is not a hostile milieu. Indeed, astrocytes, a major plurifunctional CNS glia lineage, are able to produce B cell growth factors, including BAFF, CXCL10 and CXCL13, that may contribute to supporting B cell survival. Their target antigens remain a matter of debate. Wekerle and colleagues screened recombinant cerebrospinal fluid oligoclonal immunoglobulin bands for binding to antigen libraries. They noted binding to a set of intracellular structures that hardly qualify as classic demyelinating antibody candidates (26). On the other hand, Alexander Gabibov (Russian Academy of Sciences, Moscow) reported that one of the main MS autoantigens, myelin basic protein, escapes ubiquitination control for proteasomal degradation. He proposed that this could provide an input for deregulation of degradation of autoantigens and for the antigen presentation machinery, thereby contributing to stimulation of the autoimmune processes in MS (27).

Studies by Nancy Monson (University of Texas Southwestern Medical Center, Dallas) led to the description of B cell abnormalities in MS patients that include expansion of peripheral plasmablasts, hyperproliferation to low CD40 stimulation, exaggerated IL-6 production and failure to produce IL-10. Consequently, like other human autoimmune diseases, B cell depletion has become a powerful therapy for early MS, substantially reducing relapse frequency and radiologically determined lesional load, and possibly improving neurological deficiencies. The fact that inflammatory $\mathrm{T}$ helper (Th) 1 and Th17 cells are thought to play a critical role in MS pathogenesis raises the question of whether $\mathrm{B}$ cells influence Th cell function in this disease. Monson summarized investigations using an assay system that directly tests B-T interactions ex vivo. B cells from MS patients, but not from healthy donors, were found to incite proliferation and IL-17 cytokine production by Th17 cells in response to neuroantigens and to support antigen-specific Th1 response (28). Since therapeutic B cell targeting is known to reduce Th17 response in MS patients, these observations could account, at least in part, for the beneficial effects observed. It will be important to determine the mechanisms used by B cells to support neuroantigen-driven Th17 responses. Targeting autoimmune interactions between B cell subsets and inflammatory Th cells in MS could provide novel therapeutic avenues.

\section{INSIGHT INTO PLASMA CELLS}

High-affinity antibodies provide protection against previously encountered infectious agents, and plasma cells play a chief role in their production. From $B$ cells, they differentiate under the control of a complex network of transcription factors into short-lived plasma cells that locate in extrafollicular sites of secondary lymphoid organs such as lymph node medullary cords and the splenic red pulp, and long-lived plasma cells that migrate from secondary lymphoid organs to the bone marrow and have the potential to maintain robust serological memory for decades. Key for differentiation and antibody production in plasma cells is the transcription factor B lymphocyte-induced maturation protein 1 (Blimp-1) (29). Kuo-I Lin (Academia Sinica, Taipei, Taiwan) discussed Blimp-1-mediated mechanisms in generation and maintenance of plasma cells. Her group reported that the capacity of Blimp-1 to maintain plasma cells relies on the suppression of apoptosis signalregulating kinase 1 (Ask1). Antigen-specific short-lived and long-lived plasma cells generated by immunization consistently accumulated in Ask1-deficient mice. Furthermore, her group reported that Blimp-1-mediated suppression of the transcription factor activated $B$ cell factor-1 (ABF-1) is involved in mounting plasma cell versus memory B cell response (30). By generating an inducible mouse line in which ABF-1 can be induced to translocate into the nucleus in a B cell-specific manner after immunization, Lin demonstrated that induction of ABF-1 during a T cell-dependent response leads to augmented formation of GCs and memory B cells, whereas generation of antigen-specific, antibody-secreting plasma cells was reduced after ABF-1 induction. The results suggest that ABF-1 facilitates formation of memory B cells but prevents plasma cell differentiation. Therefore, Blimp- 1 is not only crucial for driving plasma cell differentiation, but is also involved in suppression of memory B cell fate.

\section{THERAPEUTIC AVENUES}

Currently, several B cell-directed approaches are being developed for autoimmune diseases (31). Other research avenues were presented at the meeting (Figure 1). The role of Blimp-1 in SLE pathogenesis was discussed by Betty Diamond (Feinstein Institute for Medical Research, Manhasset, NY, USA). The Blimp-1 SLE risk allele exhibits 


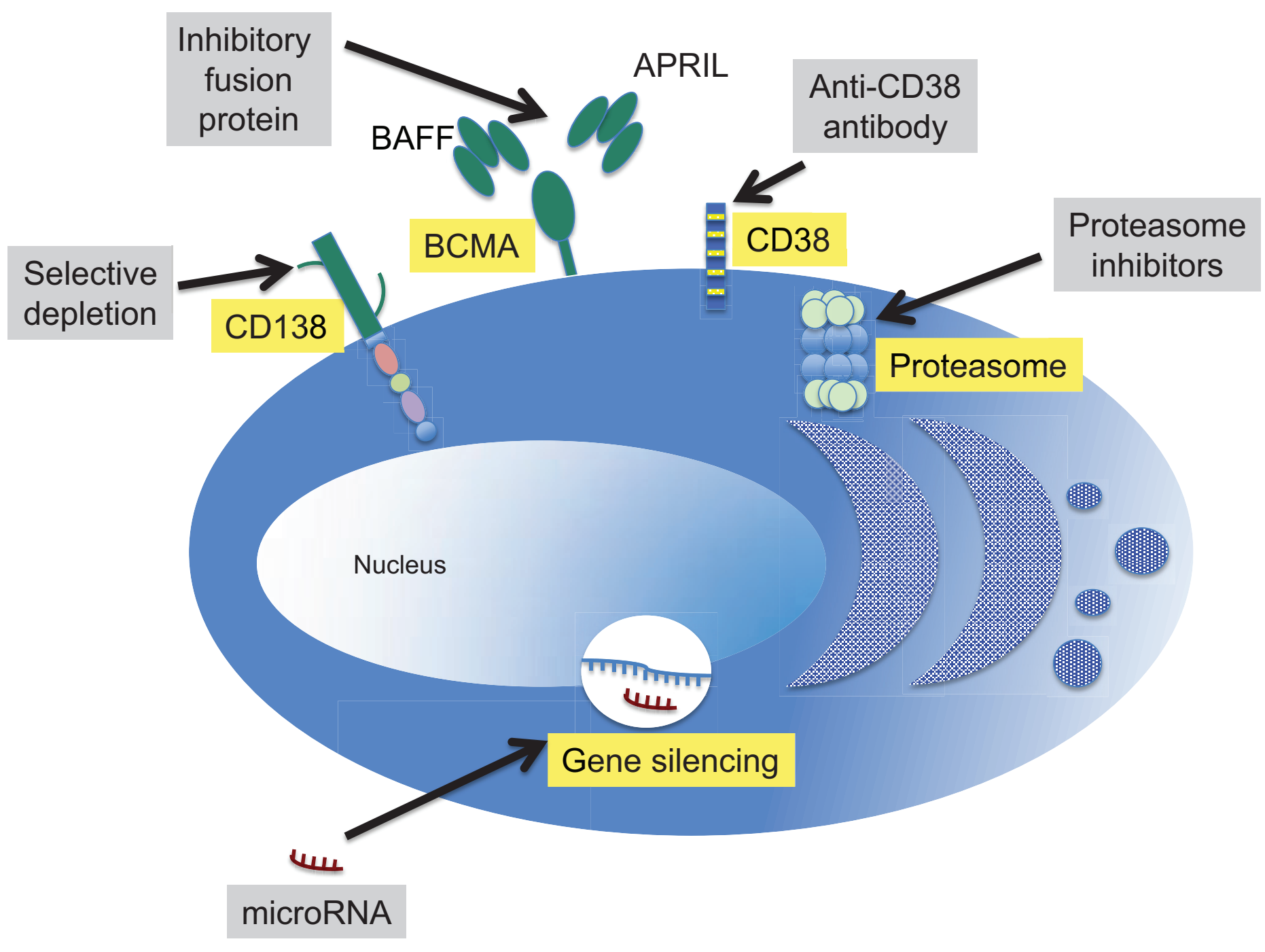

Figure 1. Potential therapeutic targets that are of potential use in autoimmune diseases. Each target is discussed in detail in the text.

low expression in dendritic cells. When C57Bl/ 6 mice are engineered to have low expression of Blimp-1 in dendritic cells, the females develop anti-DNA antibodies, which arise by somatic mutation, and glomerulonephritis. Blimp-1-deficient dendritic cells express high levels of cathepsin S, and inhibition of cathepsin $\mathrm{S}$ abrogates the disease phenotype. The view that Blimp-1 plays a role in SLE disease development is also supported by the finding that Blimp-1 siRNA eliminated anti-dsDNA antibody-producing plasma cells, reduced serum anti-dsDNA antibody levels and impeded the development of lupus (32).
Studies in murine autoimmunity models revealed that both short-lived plasmablasts or plasma cells and longlived plasma cells can secrete autoantibodies (33). Therefore, long-lived cells have emerged as a promising therapeutic target. Falk Hiepe (Charité University Hospital, Berlin, Germany) described approaches to target plasma cells in autoimmunity. Long-lived memory plasma cells secreting pathogenic autoantibodies represent key drivers of autoimmune-mediated pathology, but in contrast to short-lived plasma cells, they are independent from B cell precursors and resistant to antiproliferative agents, such as cyclophosphamide. Protected by their environment, memory plasma cells do not proliferate, but survive in dedicated niches in the bone marrow and inflamed tissues, making them refractory to conventional immunosuppression or therapy targeting B cells. A therapeutic option that largely eliminates immunological memory, including longlived memory plasma cells, consists in immunoablation with anti-thymocyte globulin, followed by autologous stem cell transplantation. Such treatment would provide the basis for development of a healthy self-tolerant immune system. However, due to the risk of severe 
infection, this immunoablative regimen is only indicated in patients with severe autoimmune disease refractory to standard-of care immunosuppression. Other studies have shown that more selective depletion of virtually all plasma cells using proteasome inhibitors can prevent pathology development in autoimmune mice. For example, Hiepe's studies demonstrated a beneficial effect of the proteasome inhibitor bortezomib in refractory SLE patients, but in contrast to findings obtained in autoimmune mice, only about $30 \%$ depletion of memory plasma cells could be achieved (34). Although proteasome inhibitors lack significant effects on B cells and T cells, additional mechanisms that act independent of plasma cell depletion might contribute to the clinical response. Another strategy would consist of targeting CD38, a cell surface protein overexpressed on multiple myeloma cells. Encouragingly, an anti-CD38 human monoclonal antibody showed encouraging efficacy and had an acceptable safety profile in clinical trials of multiple myeloma patients, selectively depleting plasma cells (35).

Limitations to plasma cell depletion include the fact that current therapeutic strategies target the whole long-lived memory plasma cell pool, even those that secrete protective antibodies. Hiepe proposed an approach to selectivelydeplete memory plasma cells that secrete pathogenic autoantibodies based on the specificity of the antibodies. It consists of labeling plasma cells with an affinity matrix composed of a $\mathrm{F}(\mathrm{ab})_{2}$ fragment of an antibody that selectively binds to plasma cells, such as anti-CD138, and the (auto) antigen specific for the (auto)antibody secreted by the plasma cells to be depleted. Results obtained in mice expressing memory plasma cells secreting antibodies against ovalbumin and chicken $\gamma$ globulin (CGG) demonstrated that an affinity matrix consisting of an anti-CD138 F(ab) fragment conjugated with ovalbumin selectively depleted plasma cells secreting anti-ovalbumin antibodies from the bone marrow, but spared anti-CGG antibody-secreting plasma cells. These encouraging results warrant preclinical studies using such an affinity matrix for selective depletion of memory plasma cells secreting pathogenic autoantibodies. Importantly, the data derived from preclinical autoimmune models and SLE patients also show that sustained therapeutic elimination of memory plasma cells additionally requires inhibition of their regeneration.

In 1900, Paul Ehrlich coined the term "magic bullet" to describe what he considered to be the affinity of a drug for its target. In immunology, it currently describes a molecule that could be directed to a target by a specific antibody and destroy the target once it reaches it. For example, monoclonal antibodies can be linked to a toxin or a cytokine for use as magic bullets. Gabibov described a recombinant immunotoxin for immunotherapy of autoreactive lymphocytes expressing MOG-specific B cell receptors. This novel immunotoxin is based on the catalytic and translocation subunits of diphtheria toxin fused with an immunodominant sequence of MOG that is recognized by autoantibodies from more than half of MS patients. The pharmacokinetics and nonspecific toxicity of the generated immunotoxin were monitored in vivo (36). Future studies should evaluate the potency of this immunotoxin as a therapeutic agent in models of MS.

\section{PERSPECTIVES}

As briefly discussed above, the B cells and Autoimmunity conference was successful in bringing together investigators from the academic and industrial sectors, and in favoring informal discussion of novel ideas. The tranquility of Sun Moon Lake provided a unique setting for this multidisciplinary event to develop various areas related to aspects of B cell biology and functions. The informal and convivial atmosphere allowed a flow of presentations and discussions from the worldwide community that ranged from basic aspects of B cell biology to clinical challenges and potential therapeutic opportunities. Sharing key findings and emerging trends, participants highlighted expectations in this dynamic field of research. Some of the recently developed approaches have expanded our ability to interrogate B cell-mediated pathways involved in autoimmune disease. They are leading to a better understanding of the complexity of autoimmune reactions in animal models and in patients. Identification of pathways that take part in the disease process is permitting the introduction of biologics with demonstrable success in clinical trials. It is hoped that the wealth of data that has been gathered and disseminated will lead to new and improved targeting strategies designed to increase objective response rates in patients with autoimmune disease. It is not unreasonable to conclude that combinations of tailored immune targeted therapeutics based on particular immunological alterations will provide patients with more successful drugs. The momentum in the field is great, and we all look forward to the seventh edition of the B cells and Autoimmunity conference.

\section{ACKNOWLEDGMENTS}

The conference was organized by Betty Diamond, Gregory Tsay, and Moncef Zouali. MZ is supported by Inserm and the University of Paris. GJT is supported by Taiwan MOST 104-2314-B-039-045. The authors thank colleagues for allowing citation of unpublished data and offer apologies for the omission of many exciting new data owing to space limitations.

\section{DISCLOSURE}

The authors declare that they have no competing interests as defined by Molecular Medicine or other interests that might be perceived to influence the results and discussion reported in this paper.

\section{REFERENCES}

1. Edwards JC, Cambridge G. (2001) Sustained improvement in rheumatoid arthritis following a protocol designed to deplete B lymphocytes. Rheumatology (Oxford). 40:205-11.

2. Walsh ER, et al. (2012) Dual signaling by innate and adaptive immune receptors is required for 
TLR7-induced B-cell-mediated autoimmunity. Proc. Natl. Acad. Sci. U.S.A. 109:16276-81.

3. Soni C, et al. (2014) B cell-intrinsic TLR7 signaling is essential for the development of spontaneous germinal centers. J. Immunol. 193:4400-14.

4. Domeier PP, et al. (2016) IFN-gamma receptor and STAT1 signaling in B cells are central to spontaneous germinal center formation andautoimmunity. J. Exp. Med. 213:715-32.

5. Zouali M, Jefferis R, Eyquem A. (1984) IgGsubclass distribution of autoantibodies to DNA and to nuclear ribonucleoproteins in autoimmune diseases. Immunology. 51:595-600.

6. Arbuckle MR, et al. (2003) Development of autoantibodies before the clinical onset of systemic lupus erythematosus. N. Engl. J. Med. 349:1526-33.

7. Permin H, Wiik A. (1978) The prevalence of IgE antinuclear antibodies in rheumatoid arthritis and systemic lupus erythematosus. Acta Pathol. Microbiol. Scand. C. 86C: 245-49.

8. Henault J, et al. (2016) Self-reactive IgE exacerbates interferon responses associated with autoimmunity. Nat. Immunol. 17:196-203.

9. Khamashta M, et al. (2016) Sifalimumab, an anti-interferon-alpha monoclonal antibody, in moderate to severe systemic lupus erythematosus: a randomised, double-blind, placebo-controlled study. Ann. Rheum. Dis. 75(11):1909-16

10. Viau M, Zouali M. (2005) B-lymphocytes, innate immunity, and autoimmunity. Clin. Immunol. 114:17-26.

11. Moon H, et al. (2015) Early Development in the Peritoneal Cavity of CD49dhigh Th1 Memory Phenotype CD4+ T Cells with Enhanced B Cell Helper Activity. J. Immunol. 195:564-75.

12. Tonti E, et al. (2009) NKT-cell help to B lymphocytes can occur independently of cognate interaction. Blood. 113:370-76.

13. Fan X, Rudensky AY. (2016) Hallmarks of Tissue-Resident Lymphocytes. Cell. 164:1198-1211.

14. Godfrey DI, Uldrich AP, McCluskey J, Rossjohn J, Moody DB. (2015) The burgeoning family of unconventional T cells. Nat. Immunol. 16:1114-23.

15. Hosseini H, et al. (2015) Phosphatidylserine liposomes mimic apoptotic cells to attenuate atherosclerosis by expanding polyreactive IgM producing B1a lymphocytes. Cardiovasc. Res. 106:443-52.

16. Tay C, et al. (2016) B-cell-specific depletion of tumour necrosis factor alpha inhibits atherosclerosis development and plaque vulnerability to rupture by reducing cell death and inflammation. Cardiovasc. Res. 111:385-97.

17. Hamze M, et al. (2013) Characterization of resident $B$ cells of vascular walls in human atherosclerotic patients. J. Immunol. 191:3006-16.

18. Natorska J, Marek G, Sadowski J, Undas A. (2016) Presence of B cells within aortic valves in patients with aortic stenosis: Relation to severity of the disease. J. Cardiol. 67:80-85.

19. Mohan C, Putterman C. (2015) Genetics and pathogenesis of systemic lupus erythematosus and lupus nephritis. Nat. Rev. Nephrol. 11:329-41.
20. Wu YY, Kumar R, Iida R, Bagavant $H$, Alarcon-Riquelme ME. (2016) BANK1 Regulates IgG Production in a Lupus Model by Controlling TLR7-Dependent STAT1 Activation. PLoS One. 11: e0156302.

21. Zouali M, Sarmay G. (2004) B lymphocyte signaling pathways in systemic autoimmunity: implications for pathogenesis and treatment. Arthritis Rheum. 50:2730-41.

22. Leslie NR, Downes CP. (2002) PTEN: The down side of PI 3-kinase signalling. Cell Signal. 14:285-95.

23. Wu XN, et al. (2014) Defective PTEN regulation contributes to B cell hyperresponsiveness in systemic lupus erythematosus. Sci. Transl. Med. 6:246ra299.

24. Gonzalez-Martin A, et al. (2016) The microRNA miR-148a functions as a critical regulator of B cell tolerance and autoimmunity. Nat. Immunol. 17:433-40.

25. Berer K, et al. (2011) Commensal microbiota and myelin autoantigen cooperate to trigger autoimmune demyelination. Nature. 479:538-41.

26. Brandle SM, et al. (2016) Distinct oligoclonal band antibodies in multiple sclerosis recognize ubiquitous self-proteins. Proc. Natl. Acad. Sci. U.S.A. 113:7864-69.

27. Belogurov A, Jr., et al. (2015) Ubiquitinindependent proteasomal degradation of myelin basic protein contributes to development of neurodegenerative autoimmunity. FASEB J. 29:1901-13.

28. Ireland SJ, Guzman AA, Frohman EM, Monson NL. (2016) B cells from relapsing remitting multiple sclerosis patients support neuro-antigenspecific Th17 responses. J. Neuroimmunol. 291:46-53.

29. Calame K. (2010) Blimp-1's maiden flight. J. Immunol. 185:3-4.

30. Chiu YK, et al. (2014) Transcription factor ABF-1 suppresses plasma cell differentiation but facilitates memory B cell formation. J. Immunol. 193:2207-17.

31. Suurmond J, Zou YR, Kim SJ, Diamond B. (2015) Therapeutics to block autoantibody initiation and propagation in systemic lupus erythematosus and rheumatoid arthritis. Sci. Transl. Med. 7:280ps285.

32. Luo J, et al. (2015) Inhibition of B lymphocyteinduced maturation protein-1 reduces the production of autoantibody and alleviates symptoms of systemic lupus erythematosus. Autoimmunity. 48:80-86.

33. Hiepe F, Radbruch A. (2016) Plasma cells as an innovative target in autoimmune disease with renal manifestations. Nat. Rev. Nephrol. 12:232-40.

34. Alexander T, et al. (2015) The proteasome inhibitor bortezomib depletes plasma cells and ameliorates clinical manifestations of refractory systemic lupus erythematosus. Ann. Rheum. Dis. 74:1474-78.

35. Sanchez L, Wang Y, Siegel DS, Wang ML. (2016) Daratumumab: a first-in-class CD38 monoclonal antibody for the treatment of multiple myeloma. J. Hematol. Oncol. 9:51.
36. Stepanov A, et al. (2016) Development of a recombinant immunotoxin for the immunotherapy of autoreactive lymphocytes expressing MOG-specific BCRs. Biotechnol. Lett. 38:1173-80.

Cite this article as: Zouali M and Tsay G. (2016) Developing connections among B lymphocytes and deregulated pathways in autoimmunity. Mol. Med. 22:705-12. 